\title{
Trabajo social y discapacidad: intervención desde el departamento de calificación del Ministerio de Salud Pública
}

Social work and disability: intervention from the department of qualification of the Ministry of Public

Health

Leila María Álava Barreiro'

David Clotario Calero Zambrano²

RECIBIDO: 30 DE ABRIL DE 2020

ACEPTADO: 20 DE JUNIO DE 2020

\section{RESUMEN}

Se considera persona con discapacidad a aquellas que tienen deficiencias físicas, mentales, intelectuales y sensoriales a largo plazo, que, al interactuar con diversos contextos, puedan impedir su participación plena y efectiva en la sociedad, por ello, el objetivo de esta investigación consistió en analizar la intervención del Trabajador Social para el otorgamiento del carnet de discapacidad en el departamento de calificación del Ministerio de Salud Pública del Ecuador. Se utilizó una metodología

\footnotetext{
1 Magister en Educación y Desarrollo Social, Licenciada en Trabajo Social, Docente, Universidad Técnica de Manabí, Ecuador; lalava@utm.edu.ec, ORCID: https://orcid.org/0000-0001-9494-3402 Google Scholar: https://scholar.google.es/citations?hl=es\&user=DBOnsSWAAAAJ

2 Egresado de Trabajo Social, Universidad Técnica de Manabí, dcalero6358@utm.edu.ec, ORCID: https://orcid.org/0000-0002-5051-008X ,
} 


\section{2}

de tipo no experimental, apoyado en el método descriptivo, bibliográfico; se aplicó el método lógico científico: inductivo-deductivo con enfoque cualitativo, lo que permitió cumplir con el objetivo planteado. Como resultado se tiene la intervención del profesional en Trabajo Socia la cual consiste en realizar la valoración social del usuario, en ésta se abordan factores socioeconómicos, individual y familiar, por medio de la matriz de baremo, la misma que permite otorgar una calificación que sumada a la del diagnóstico final del médico, se determina si aplica para otorgar o no el carné de discapacidad. A modo de conclusión se indica que la intervención del profesional en Trabajo Social, es fundamental, por ser un agente de cambio e inclusión social, el cual ejecuta actividades y procesos en búsqueda de mejoras en usuarios con discapacidad, disminuyendo barreras que limitan de forma directa su participación en sociedad.

Palabras clave: personas con discapacidad, trabajo social, Ministerio de Salud Pública del Ecuador

\section{ABSTRACT}

People with disabilities are considered to be those who have long-term physical, mental, intellectual, and sensory deficiencies that, by interacting with various contexts, may impede their full and effective participation in society, therefore, the objective of this research was to analyze the intervention of the Social Worker for the granting of the disability card in the qualification department of the Ministry of Public Health of Ecuador. The methodology used was of a non-experimental type, supported by the descriptive, bibliographic method; The scientific logical method was applied: inductive-deductive with a qualitative approach, a methodology that enabled the stated objective to be met. As a result, emphasis is placed on the intervention of the professional in Social Work, which consists of carrying out the social assessment of the user, in which socioeconomic, individual and family factors are addressed, for which the baremo matrix is used, which allows granting a qualification that added to the one of the final diagnosis of the doctor determines a qualification to grant or not the disability card. The intervention of the professional in Social Work is essential, as it is an agent of change and social inclusion, which executes activities 
and processes in search of improvements in users with disabilities, reducing barriers that directly limit their participation in society.

Keywords: people with disabilities, social work, Ministry of Public Health of Ecuador

\section{Introducción}

El Trabajo Social tiene especial implicación en las personas con discapacidad dado que esta profesión va dirigida a velar por principios como la dignidad, la autonomía, la no discriminación, fomentando la participación e inclusión, basado en la igualdad de oportunidades que involucren una verdadera inclusión social, y no una limitación dada la condición de las personas. En este sentido, (Lorna, 2014) manifiesta que "el Trabajador Social interviene en aquellas dimensiones sociales que determinan las condiciones de vida de la persona, para eliminar o minimizar barreras sociales, o para generar, fortalecer o proveer facilitadores sociales del mismo".es decir que el profesional trabaja de forma incluyente en aquellos usuarios que requieran una intervención eficaz, como en el caso de las personas con discapacidad.

Es importante indicar que hablar de discapacidad involucra diferentes variantes, pero de forma general hace énfasis a deficiencia en la persona quien la posee, de acuerdo a la Organización Mundial de la Salud (2011) "más de mil millones de personas es decir, un $15 \%$ de la población mundial están aquejadas por la discapacidad en alguna forma. Tienen dificultades importantes para funcionar entre 110 millones (2,2\%) y 190 millones (3,8\%) personas mayores de 15 años". Es evidente indicar que esta cifra es superior a las estimaciones previas de la Organización Mundial de la Salud, ya que ha existido un incremento de la población mundial y con ella nuevos casos de discapacidad que ocurren durante el envejecimiento poblacional. En esta situación, la salud entendida como producto de las condiciones sociales y biológicas y a la vez como productora de mejoras que permiten el desarrollo integral a nivel individual y colectivo.

En ecuador como parte del reconocimiento a esta problemática de salud pública, de acuerdo a la misión toda una vida (2018) "mediante Decreto Ejecutivo No. 63 , se crea la Secretaría Técnica de Discapacidades (SETEDIS), a fin de que impulse entre otras estrategias el Gobierno accesible, la investigación técnica y científica, la marca 


\section{4}

de productos elaborados por personas con discapacidad". La creación de esta secretaria impulsaría un adecuado ambiente de inclusión en las personas con esta problemática, Por consiguiente, la discapacidad es un fenómeno complejo que refleja una interacción entre las características del organismo y las características de la sociedad en la que vive. Por ello el objetivo de esta investigación consiste en analizar la intervención del trabajador social para el otorgamiento del carné en el departamento de calificación de discapacidad del Ministerio de Salud Pública del Ecuador

\section{Desarrollo}

La constitución de la República del Ecuador, en sus artículos 47, 48 y 49 tienen como principios garantizar la protección y el desarrollo integral para las personas con discapacidad y sus familiares de acuerdo al (Reglamento a la Ley Organica de Discapacidades, 2017), en el artículo 1 define a la persona con discapacidad de la siguiente manera.

Se considera persona con discapacidad a toda aquella que, como consecuencia de una o más deficiencias físicas, mentales, intelectuales 0 sensoriales, con independencia de la causa que lo hubiere originado, ve restringida permanentemente su capacidad biológica, psicológica y asociativa para ejercer una o más actividades esenciales de la vida diaria.

Ecuador en el año 1999 firmó la Convención Interamericana para la Eliminación de todas las formas de discriminación contra las personas con Discapacidad, esta acción fue ratificada en 2004, en ella se reconoce a estas personas como sujeto de derechos al igual que otras personas. En este sentido, de forma inclusiva en el país, se ha implementado acciones que permitan la intervención de personas con discapacidad, mediante la aplicación de políticas que hagan gestión, coordinación e implementación de ayudas a este grupo de atención prioritaria, para ello se ha creado el Programa Misión Las manuelas, quien entre otros aspectos:

... emprenderá la actualización de información, provisión de ayuda técnica, pensiones asistenciales y vivienda, así como servicios de salud, inclusión laboral, educativa y comunitaria. La garantía de este derecho se logrará con acciones coordinadas y articuladas entre: la Secretaría Técnica del Plan Toda una Vida, el 
Ministerio de Salud Pública, el Consejo Sectorial Social, el Consejo Nacional de Igualdad de Discapacidades y el Ministerio de Inclusión Económica y Social. (Plan Nacional de Desarrollo, 2017, p. 68)

La Misión Las Manuelas busca garantizar la atención integral de las personas con discapacidad y la de sus núcleos familiares. Para esto se considera importante promover acciones que permitan a las personas con discapacidad alcanzar su autonomía para que puedan tomar decisiones sobre aquellos temas específicos que afectan su desarrollo

\section{Tipos de discapacidad}

De acuerdo a los diferentes tipos de discapacidad, el Ministerio de Salud Pública (MSP, 2015) menciona que "Los tipos de discapacidad son: auditiva, física, intelectual, lenguaje, psicosocial y visual; en tanto, cada una de estas puede manifestarse de diferentes maneras y en diferentes grados". Esta entidad asumió la competencia de la calificación y registro de personas con discapacidad desde el año 2013, centrando su atención en el otorgamiento del carné el cual es emitido en los centros de salud autorizados a nivel nacional.

Para profundizar en los casos de calificación más frecuentes se mencionan los tipos de discapacidad de manera ordenada: Auditiva, de acuerdo a la Guía de Apoyo Técnico Pedagógico: Necesidades Educativas Especiales en el Nivel de Educación Parvulario (2016, p.7) menciona que es una " dificultad que presentan algunas personas para participar en actividades propias cotidiana, que surge como consecuencia de la interacción entre una dificultad específica para percibir a través de la audición los sonidos del ambiente y dependiendo del grado de pérdida auditiva". Este tipo de discapacidad limita la interacción entre las personas para una comunicación adecuada, sin embargo, no implica que con la perdida de la audición se debe airar del contento a quien la padece.

Continuando con la siguiente discapacidad se encuentra la Física, en ella (Acera, 2020) menciona que ésta "limita o impide el desempeño motor de la persona. Las causas de la discapacidad física muchas veces son congénitas o de nacimiento. También pueden ser causadas por lesión medular en consecuencia de accidentes (paraplejía) o problemas del organismo (derrame cerebral)". Es decir que se puede 


\section{6}

padecer en cualquier momento este tipo de discapacidad por los criterios antes mencionados. En el siguiente orden se encuentra la intelectual, esta "es un funcionamiento intelectual situado significativamente por debajo del promedio, que está presente desde el nacimiento o la primera infancia y que causa limitaciones para llevar a cabo las actividades normales de la vida diaria". Por ello Las personas afectadas tienen un funcionamiento intelectual significativamente bajo, lo que implica su capacidad de realizar actividades cotidianas. A continuación, tenemos la discapacidad de Lenguaje, de acuerdo al Servicio de información sobre discapacidad (2020) menciona que "Se refiere a personas con deficiencias de comprensión y/o elaboración del lenguaje, la producción y/o emisión del habla, y los trastornos de la voz". Este tipo de discapacidad afecta directamente la forma de comunicarse de modo sonora, empleando el uso de un lenguaje complementario para una adecuada comunicación. En concordancia con los tipos anteriores, continúa la discapacidad psicosocial, para un acercamiento a la definición, se menciona a (Guillen, 2018), quien la define como "personas con diagnóstico de trastorno mental que han sufrido los efectos de factores sociales negativos, como el estigma, la discriminación y la exclusión". Comprende aquellas personas quienes han sufrido algún tipo de alteración mental causando un impacto considerable para un adecuado comportamiento en la sociedad. Y por último se encuentra la visual, la cual hace relación a "una anomalía o dificultad del ojo o los ojos, de sus estructuras que trae como consecuencia una visión menor de la normal y precisa en ocasiones medios de ayuda para la interacción entre las personas" (EcuRed, 2018). En este sentido, la limitación de actividad y también las restricciones de la participación son un componente que tienden a dificultar la interacción social de las personas con algún tipo de discapacidad.

En relación al Trabajo Social inmerso en el campo de la salud éste se involucra como un ente especializado que implementa y ejecuta políticas sociales, que se orienten a garantizar los derechos de las personas quienes requieran una intervención. Así lo señala, (Nucci, Crosetto, Bilavcik y Miani, 2018) "Trabajo Social es una de las profesiones que forma parte de los equipos interdisciplinarios de salud, o que en forma disciplinaria tiene a su cargo la vinculación con sujetos que demandan atención". Es decir que se generaliza al profesional como complemento de ejecutar 
acciones en búsqueda del bienestar de los usuarios. En este escenario (Abreu Velázquez y Mahtani Chugani, 2017) mencionan que "los trabajadores sociales sanitarios en el primer nivel asistencial resultan claves para el abordaje de problemas de salud complejos donde se asocian patologías crónicas y necesidades sociales". Es decir, este profesional esta con plenos conocimientos de su accionar, brindando un adecuado y oportuno proceso para el bienestar de las personas, para lo cual se le deben incluir roles principales en los centros de primer nivel, sin embargo, (Díaz, 2013) manifiesta:

El Trabajo Social se incluye en la estructura organizativa de las entidades, sin embargo, en la normativa reguladora del funcionamiento de los centros, no se explicitan las funciones de trabajadores/as sociales, salvo que junto a otros profesionales pueden hacerse cargo de los servicios de apoyo psicosocial.

El rol que desempeña el profesional en los centros de primer nivel de salud, debe ser normativos, en el cual estén establecidas las acciones que regulen, y orienten las actividades que involucren el ejercicio profesional del trabajador social y éste a su vez pueda desempeñar mejor su función al servicio de las personas con discapacidad.

\section{Método}

Para la ejecución de esta investigación se realizó un tipo de estudio no experimental, en el cual se utilizaron los métodos descriptivo y bibliográfico; así mismo se aplicó el método lógico científico: inductivo-deductivo con enfoque cualitativo, metodología que permitió cumplir con el objetivo planteado.

\section{Resultados}

En esta investigación se considera la intervención del profesional en Trabajo Social para el proceso de calificación previo a la obtención del carné de discapacidad por parte de los usuarios, por ello, se mencionan los procedimientos que los usuarios deben realizar en donde se involucra el profesional para dicho proceso.

El usuario que requiera la calificación debe disponer de la cédula de ciudadanía en conjunto con los exámenes médicos complementarios y adicional el informe 


\section{8}

médico-especialista, estos documentos deberán tener el respectivo sello y firma. Es importante indicar que solo son admitidos los documentos que sean generados en la red pública de salud y no de forma particular. Para el proceso de calificación, el usuario deberá realizar los siguientes procedimientos:

1. Llamar al número telefónico 171 opción 4 para solicitar una cita médica de evaluación bio-psicosocial esta acción la puede realizar un familiar de la persona con discapacidad.

2. Asistir al establecimiento de salud de primer Nivel autorizado con 30 minutos antes de la hora establecida con los requisitos correspondientes.

3. Obtener el carné de persona con discapacidad en el establecimiento de Salud donde lo evaluaron en un plazo aproximado de 15 días posterior a la calificación y en otros de manera inmediata.

La intervención del profesional en Trabajo Social consiste en realizar la valoración social del usuario solicitante, en ésta se abordan factores socioeconómicos, para lo cual se utiliza la matriz de valoración social de Baremo actualizada, ésta consiste entre otros aspectos a identificar información relevantes a la situación familiar, recursos económicos situación laboral y profesional, nivel cultural, y situaciones sociales del entorno, en ésta permite otorgar una calificación máxima de 15 puntos, que serán sumadas al diagnóstico final por parte del médico calificador. Se menciona que en el caso de usuarios que no pueden ser movilizados por su condición de discapacidad, los profesionales calificadores: en médico, psicólogo y trabajador social, deben realizar una visita domiciliaria en la cual se ejecutará en la valoración.

Cuando el usuario, luego de la valoración, se considere necesaria la ayuda técnica, el trabajador social suscribe una ficha de preinscripción de ayudas técnicas, la cual consiste en identificar las necesidades que requiere el usuario con implementos para mejorar su condición, previo a lo estipulado por el medico calificador.

Resulta oportuno manifestar que el trabajador social implementa acciones de intervención durante y después de la valoración, en aquellos casos donde se requiera el respectivo seguimiento a usuarios calificados, con el control y entrega periódica de los implementos de ayudas técnicas. 


\section{Discusión}

El trabajo social involucra diferentes acciones que son importantes en las personas con discapacidad, al ser evidente la necesidad que padecen estas personas quienes en gran proporción, son vulneradas, de acuerdo lo expuesto por (Rodriguez, 2013) manifiesta que "las personas con discapacidad son especialmente vulnerables a la exclusión social, por diversos factores, entre otros la carencia de los bienes imprescindibles para ejercer plenamente sus derechos de ciudadanía". Es decir que existen barreras en las personas que por su condición afectan directamente su participación, en distintos eventos sociales, políticos y laborales, por ende, es importante la intervención del Trabajador Social, quien con su accionar, busca minimizar estas situaciones. Así lo manifiesta (Universidad de Almería, 2020) quien indica que:

El trabajador/a social es el profesional que facilita cambios personales y sociales, mediante tareas de prevención, atención, gestión, orientación, mediación, apoyo y dinamización social, con el objetivo de mejorar la calidad de vida de las personas y los grupos. Este profesional también está presente en la formulación de políticas de bienestar social.

Es evidente que el accionar del trabajador social engloba distintos sectores sociales, especialmente a quienes están vulnerados en derechos que impliquen su participación y no se limite por su condición de discapacidad, esta situación generalmente se realiza desde diferentes instituciones que tienen como objetivo intervenir con personas que presentan discapacidades como el Ministerio de salud pública, desde allí es fundamental la intervención del profesional quien entre otros aspectos considera la importancia socioeconómica, afectiva y familiar para una adecuada gestión, tal como lo señala (Camargo Rojas, Gómez Serna y Molina Murcia, 2019) La condición socioeconómica de las familias es una variable que se aproxima al estudio de la exclusión social de la población con discapacidad.

Es importante la gestión que realiza el profesional en Trabajo Social durante la valoración, previo a la calificación, pues aquí se consideran aspectos esenciales desde el ámbito social, así lo menciona (El Comercio, 2018) "se tomarán en cuenta parámetros como 'participación social y contexto'. Esto abarca las condiciones de vida de la persona". Es decir, aquí se plasma la importancia en la intervención del 


\section{0}

trabajador social, quien posee los conocimientos necesarios para intervenir en el contexto social de las personas con discapacidad.

\section{Conclusión}

La intervención del profesional en Trabajo Social durante la calificación previo a la obtención del carné de discapacidad, es fundamental, por ser un agente de cambio e inclusión social, el cual ejecuta actividades y procesos en búsqueda de mejoras en usuarios con discapacidad, disminuyendo barreras que limitan de forma directa su participación en sociedad.

El ministerio de salud pública del ecuador, como ente rector de ejecución en la calificación para el otorgamiento del carné de discapacidad, permiten la participación del profesional en Trabajo Social de forma activa, el cual aplica los conocimientos de manera complementaria en conjunto con el equipo interdisciplinar quienes, a su vez, realizar los procedimientos que estipula ese ministerio.

El diagnóstico que presenta el trabajador social durante la calificación del usuario, es importante para complementar las ayudas respectivas por parte del estado, quien, por medio de esta calificación, es quien considera si es apropiado o no el otorgamiento de ayudas técnicas por cuanto su función es determinar la situación socioeconómica del usuario objeto de intervención.

\section{Referencias}

Abreu Velázquez, M., Mahtani Chugani, V. (11 de Mayo de 2017). Las funciones de los trabajadores sociales sanitarios en atención primaria de salud: evolución, redefinición y desafíos del rol profesional. Cuadernos de Trabajo Social, 31 (2), 356. doi:http://dx.doi.org/10.5209/CUTS.54560

Acera, M. (8 de Abril de 2020). Deuto Salud. Obtenido de https://www.deustosalud.com/blog/teleasistencia-dependencia/conceptodiscapacidad-diferencias-entre-discapacidad-deficiencia 
Auditiva, G. (18 de Febrero de 2016). Mineduc. Obtenido de https://especial.mineduc.cl/wpcontent/uploads/sites/31/2016/08/GuiaAuditiva.pdf

Camargo Rojas, D. A., Gómez Serna, E. A., Molina Murcia, P. S. (Octubre de 2019). Condición física relacionada con la salud y situación socioeconómica de niños y jóvenes con discapacidad intelectual de los colegios distritales de la ciudad de Bogotá. Siglo Cero, 50(272), 56. doi:http://dx.doi.org/10.14201/scero20195043959

Discapacidad, S. d. (17 de Enero de 2020). SID. Obtenido de https://sid.usal.es/colectivos/discapacidad/discapacidades-sensorialesexpresivas/deficiencias-lenguaje-habla-voz.aspx

EcuRed. (2018). Ecured. Obtenido de https://www.ecured.cu/Discapacidad_visual

El Comercio. (19 de Diciembre de 2018). Diario el Comercio. Obtenido de https://www.elcomercio.com/actualidad/carne-discapacidad-conadiscalificacion-evaluacion.html

Guillen, C. (08 de Agosto de 2018). Bajo Lupa. Obtenido de https://documenta.org.mx/blog-documenta/2018/08/08/personas-condiscapacidad-psicosocial-invisibles-pero-discriminadas/

Lorna, S. (27 de Diciembre de 2014). Issuu. Obtenido de https://issuu.pdfdownloader.com/print.php?documentld=14122802281874cdcc6588deea51ad9b4afa8b5aeffb\&count $=17$

MSP. (17 de Marzo de 2015). Ministerio de Salud Publica . Obtenido de https://www.salud.gob.ec/calificacion-o-recalificacion-de-personas-condiscapacidad-2/

Nucci, N., Crosetto, R., Bilavcik, C., y Miani, A. (5 de Marzo de 2018). La intervención de Trabajo Social en el campo de la salud pública. Conocimientos, 1 (2), 15. Recuperado el 28 de Marzo de 2020, de https://revistas.unc.edu.ar/index.php/ConCienciaSocial/

Plan Nacional de Desarrollo. (26 de Octubre de 2017). Planificacion . Obtenido de https://www.planificacion.gob.ec/wpcontent/uploads/downloads/2017/10/PNBV-26-OCTFINAL_OK.compressed 1.pdf 


\section{2}

Reglamento a la Ley Organica de Discapacidades . (27 de Octubre de 2017).

Consejo Nacional de discapacidades . Obtenido de

https://www.consejodiscapacidades.gob.ec/wp-

content/uploads/downloads/2017/1 1/Regalamento-lod-decre_-194.pdf

Rodriguez, J. M. (2013). La (con)ciencia del Trabajo Social en la discapacidad:

Hacia un modelo de intervención social basado en derechos 1. Portularia, 10.

Obtenido de

https://pdfs.semanticscholar.org/5b24/a6add3e3d8739123ee931076c43ee9e 2ef40.pdf

Salud, O. M. (2011). Informe Mundial de la Salud. Ginebra: Avenue Appia.

Toda Una Vida . (2018). Toda Una Vida. Obtenido de

https://www.todaunavida.gob.ec/

Universidad de Almería. (28 de Enero de 2020). Universidad de Almería. Recuperado el 15 de Abril de 2020, de http://cms.ual.es/UAL/estudios/grados/grado6810 